Original Paper http://ajol.info/index.php/ijbcs http://indexmedicus.afro.who.int

\title{
Impact des paramètres de sondage sur la gestion durable des forêts : cas des Forêts communales de l'Est-Cameroun
}

\author{
Armel Emmanuel BAYOI ${ }^{1}$, Elvis Mouyakan A MOUMBOCK ${ }^{1,2}$ et \\ Mbezele Junior Yannick NGABA ${ }^{3 *}$ \\ ${ }^{I}$ Ministère de la Recherche Scientifique et de l'Innovation (MINRESI), Yaoundé, Cameroun. \\ ${ }^{2}$ Département de Foresterie, Faculté d'Agronomie et des Sciences Agricoles (FASA), Université de Dschang, \\ B.P. 222 Dschang, Cameroun. \\ ${ }^{3}$ College of Natural Resources and Environment, Northwest A\&F University, Yangling 712100, Shaanxi, China. \\ ${ }^{*}$ Corresponding author; E-mail: ngabajunior@yahoo.fr; Tel: +86 18305996238
}

Received: 23-09-2020

Accepted: 26-02-2021

Published: 28-02-2021

\section{RÉSUME}

Parmi les plus vastes d'Afrique, les forêts du Cameroun sont des écosystèmes uniques qui jouent un rôle important pour son économie, la population locale et l'environnement. Toutefois, qu'il a indéniablement besoin d'utiliser ses ressources naturelles précisément la ressource ligneuse pour son développement. La déforestation fait peser une véritable menace sur cette biodiversité, ce qui impose de mettre des mesures appropriées afin de garantir sa gestion durable. La présente étude visait à évaluer les plans d'aménagement des forêts communales de l'Est Cameroun. Un total de 08 forêts communales (FC) sur 14 ont fait l'objet de cette étude, car ayant des plans d'aménagement valides. Il ressort de l'analyse des données que de chaque FC échantillonnée respectent les dispositions réglementaires. Le taux de sondage (TS) varie en fonction du coefficient de variation (CV) et de l'erreur souhaitée (ES) avec un impact considérable dans le nombre de parcelles échantillonnées et la longueur totale des layons d'inventaire. L'évolution de la surface à échantillonner est fonction du CV. Mais, il n'existe pas une différence significative des surfaces à échantillonner lorsque le $\mathrm{CV}$ varie de 150 à 350 et l'erreur fixée à 20 et à 25 . L'équidistance diminue en fonction du CV et quel que soit l'ES que le concessionnaire décide d'adopter. Afin de pallier à ce problème, la prise en compte des limites de l'aménagement et la réalisation des inventaires d'aménagement dans chaque type de forêt doivent être analysées et permettre ainsi de déterminer les DME appropriés pour chaque type de $\mathrm{FC}$ au Cameroun.

(C) 2021 International Formulae Group. All rights reserved.

Mots clés : Forêt communale, plan d'aménagement, paramètre d'aménagement, Est-Cameroun.

\section{Impact of survey parameters on sustainable forest management: case of the Communal Forests in East Cameroon}

\begin{abstract}
Among the largest in Africa, Cameroon's forests are unique ecosystems that play an essential role in its economy, local population and environment. However, as it undeniably needs to use its natural
\end{abstract}


resources precisely the wood resource for its development, deforestation poses a real threat to this biodiversity which requires appropriate measures to ensure its sustainable management. This study aimed at assessing the management plans of communal forests in East Cameroon. A total of 08 communal forests (CF) out of 14 were the subject of this study as they have valid management plans. The analysis of the data shows that each FC sampled respects the regulatory provisions. The sampling rate (TS) varies according to the coefficient of variation $(\mathrm{CV})$ and the desired error (ES) with a considerable impact on the number of plots sampled and the total length of the inventory ride. The evolution of the area to be sampled is a function of the CV. However, there is no significant difference in the areas to be sampled when the CV varies from 150 to 350 and the error set at 20 and 25. The equidistance decreases according to the CV and regardless of the ES that the dealer decides to adopt. In order to overcome this problem, taking into account the limits of management and carrying out management inventories in each type of forest must be essential and thus enable the determination of appropriate DMEs for each type of FC in Cameroon.

(C) 2021 International Formulae Group. All rights reserved.

Keywords: Communal Forest, Management plan, Management parameter, East-Cameroon.

\section{INTRODUCTION}

La gestion des ressources forestières est une préoccupation majeure pour toutes les parties prenantes de la filière bois (FAO, 2003). Pendant plusieurs décennies, la richesse de la forêt tropicale a fait croire qu'elle était inépuisable et son exploitation s'est faite de façon irrationnelle et anarchique, sans se soucier de la réglementation et de la pérennité des espèces exploitées (COMIFAC, 2007). Il a pour objectif principal de garantir la gestion durable des forêts tout en obtenant un rendement soutenu de la production (OIBT, 2006). L'exploitation forestière contribue en moyenne $6 \%$ au PIB et environ à $16,6 \%$ aux exportations annuelles depuis l'an 2000 (FAO, 2018). Les forêts communales au Cameroun sont des forêts du domaine permanent de l'État ayant fait l'objet d'un décret de classement pour le compte des Communes qui en ont fait la demande et sa gestion se fait sur la base d'un plan d'aménagement (MINFOF, 2015; Cuny, 2011). Le document de stratégie pour la croissance et l'emploi (DSCE) «Cameroun à l'horizon $2035 »$ dans son sous-programme de développement communautaire et participatif par exemple, prévoyait $75 \%$ des forêts communales auront un plan d'aménagement élaboré et approuvé en 2015. En outre, les modalités de gestion de ses terroirs limitent les ressources financières des communes, nécessaires pour supporter les coûts relativement élevés de mise en œuvre des prescriptions de gestion durable contenues dans les plans d'aménagement, ce qui augmente les risques de dégradation (Djiegni et al., 2016). Par ailleurs, treize ans après la création des forêts communales au Cameroun, il apparaît que ce mode particulier de gestion forestière décentralisée n'a pas encore atteint ses objectifs. L'implication des populations dans l'aménagement reste largement superficielle et la répartition des revenus économique générée par l'exploitation des ressources forestières suscite généralement des tensions sociales vives (Lescuyer et al., 2016).

Malgré les dispositions nationales et internationales applicables FC, ces forêts continuent d'être exploitées de manière anarchique et non soutenue, ce qui entraîne : leur dégradation. On se demande dès lors si la méthodologie d'estimation des paramètres de sondage proposée par l'administration ne favorise pas la gestion durable des ressources ligneuses des forêts communales. Il est ainsi important de savoir si l'estimation des paramètres de sondage contenus dans les plans d'aménagement est cohérente et si l'implémentation de ces mesures garantie une reconstitution tangible et réelle des essences exploitées. L'objectif général de la présente étude est de contribuer à la gestion durable des FC de l'Est Cameroun au travers de l'efficacité des plans d'aménagement étudiés. Spécifiquement, il s'agissait de : 1) Identifier les forêts communales; 2) Analyser chacune 
des FC; 3) Démontrer les limites ou incohérences de la norme dans l'estimation des paramètres du plan de sondage des FC étudiées ; 4) Proposer une meilleure technique d'estimation des paramètres de sondage au Cameroun. La revue de littérature nous a permis d'établir les hypothèses suivantes: $100 \%$ des forets communales de l'EstCameroun ont des plans d'aménagement valides et implémentent les exigences prévues dans le cahier de charge; les forêts communales étudiées sont toutes des forêts aménagées; l'estimation du potentiel ligneux dans les FC a été mal réalisée entraînant des pertes de devises importantes tant pour le concessionnaire que pour l'État; la formule proposée par Durrieu de Madron et Fourni (1997) est favorable à une meilleure estimation des paramètres d'aménagement.

\section{MATÉRIEL ET MÉTHODES \\ Localisation de la zone d'étude}

La zone de l'Est Cameroun couvre quatre départements à savoir : Lom et Djerem, Kadey, Haut Nyong et Boumba et Ngoko (Figure 1). Elle s'étend sur une superficie de $109002 \mathrm{~km}^{2}$ avec une densité de 6,53 habitants au $\mathrm{km}^{2}$ et a pour chef-lieu de région Bertoua (Mertens et al., 2007). Elle compte 14 forêts communales. Le climat de la région de l'Est subit dans son ensemble l'influence du climat équatorial de type guinéen classique qui se caractérise par des températures élevées et constantes et des précipitations plus abondantes que dans les régions balnéaires. La hauteur moyenne des pluies atteint $1700 \mathrm{~mm}^{3}$ par an et la température moyenne se situe autour de $25^{\circ} \mathrm{C}$. Les mois les moins pluvieux sont ceux de décembre, janvier et février tandis que le mois le plus pluvieux est septembre comme l'indique la Figure 2. Trois types de sols dominants peuvent être distingués: les sols ferrallitiques rouges dérivés des roches métamorphiques, les sols ferrallitiques rouge dérivé des roches basaltiques et hydromorphes (PFBC, 2006). Au Nord, on note la présence des collines à pentes assez fortes avec des sommets culminant entre $650 \mathrm{~m}$ et $480 \mathrm{~m}$. Au
Sud, on observe des vallées fluviales (OIBT, 2005 ; PFBC, 2006).

Les forêts de la région de l'Est sont arrosées par un important réseau de cours d'eau dont la plupart sont des affluents de la rivière est la Boumba et de la Sanaga (MINFOF, 2015). Cette région est dominée par trois grandes zones de la végétation: la zone forestière au sud de la région couvre les départements de la Boumba et Ngoko, du Haut-Nyong et partiellement ceux de la Kadey et du Lom et Djerem; la zone de transition au centre qui couvre les localités situées autour de Bertoua et de Ndélélé et la zone de savane qui englobe toute la partie du nord (Ngoufo et al., 2012). Ces types de forêts indiquent que les forêts communales de l'Est se situent dans une zone de transition. Dans les zones de Lom et Djérem et la Kadey, l'on retrouve le plus les espèces de la famille des Méliacées, des Sterculiacées et les Ulmacées. Parmi les principales essences rencontrées, on a, entre autres : l'ayous (Triplochyton scleroxylon), le fraké (Terminalia superba), le sapelli (Entandrophragma cylindicum), le padouk rouge (Pterocarpus soyauxii), le tali (Erytrophleum ivorense), le kotibé (Nesogordinia papaverifera), le kossipo (Entandrophragma candolei) et le dibetou (Lovoa trichilioides) (Gonmadje et al., 2015).

Les formations végétales sont inondées pendant la saison des pluies, mais l'évacuation des eaux se fait rapidement permettant ainsi leur assèchement. Plusieurs espèces de mammifères peuvent être observées dans la zone: l'éléphant (Loxodonta africana cyclotys), le buffle (Syncerus caffernanus), le chimpanzé (Pan troglodydes), le gorille (Gorilla gorilla), la panthère (Panthera pardus); le sitantunga (Trogolaptus spekii). Malheureusement, cette faune est menacée à cause d'un braconnage intensif surtout dans la partie du sud du massif forestier (Bobo, 2002 ; MINFOF, 2011). Les grands groupes ethniques de la population sont dominés par les Makas, les Gbaya de la famille des Bantous et les pygmées. Ils pratiquent généralement comme activités l'agriculture, la chasse et la cueillette ou travaillent pour des 
compagnies forestières et d'autres sociétés industrielles (FAO, 2007).

\section{Collecte des données}

État des lieux des FC étudiées et échantillonnage

L'étude a été menée de mai à juillet 2018. Elle repose sur les plans d'aménagement (PA) de $14 \mathrm{FC}$, reparties dans les quatre départements de la région de l'Est Cameroun. Un total de 09 FC étaient en activité, 08 possédaient un PA valide et ont constitué l'échantillon de la présente, soit un taux d'échantillonnage de $57,1 \%$. La première étape a consisté à entrer en possession des PA et de faire une évaluation détaillée afin d'effectuer l'état des lieux de ses FC aménagées. La base de données ainsi obtenue a été regroupée en deux grandes classes : (1) l'état de la forêt (date, décret de classement, superficie, taux de sondage, équidistance, affectation des terres et typologies); (2) les paramètres d'aménagement: Accroissement Moyen Annuel (AMA), rotation, diamètre minimum d'exploitation/AME, superficie des parcelles, productivité, superficie sondée, nombre de parcelles et longueur des layons.

\section{Détermination de la typologie en fonction du statut des plans d'aménagement en fonction du zonage et l'impact du non- respect des normes}

La détermination de la typologie s'est faite sur la base des données issues des plans d'aménagement conçus au préalable et de la technique d'évaluation du CENADEFOR (1983). Pour la détermination de l'impact du non-respect des normes, il a été question d'identifier les paramètres liés au calcul du taux de sondage et équidistances et la longueur des layons d'inventaire sur la base des données initiales.

\section{Démontrer les limites ou incohérences de la norme dans l'estimation des paramètres du plan de sondage des FC étudiées}

Les paramètres de sondage de chaque FC ont été extraits des plans d'aménagement puis rangés dans un tableau. Cette analyse nous a permis de démontrer les incohérences qui existent entre les paramètres de sondage à travers les variations observées.

Stratégie de gestion et d'estimation des paramètres de sondage des FC par l'administration

La stratégie proposée a été établie sur la base de la détermination des limites des aménagements forestiers au Cameroun dans l'estimation des paramètres de sondage des FC. En fixant les coefficients de variation et l'erreur souhaitée, De Madron et Fourni propose une autre stratégie d'estimation des paramètres de sondage.

\section{Analyse des données}

Les données collectées ont été triées et classées dans un tableur Microsoft Excel (2016). La détermination des paramètres d'aménagement a été faite à l'aide des formules de Durrieu de Madron et Forni (1997) par l'usage de certains paramètres fixes (coefficient de variation) pour nous permettre de voir le comportement des autres paramètres du plan de sondage et déduire son impact direct sur les résultats d'inventaire.

$E=(T \times C V) / \sqrt{N}$

Où $\mathrm{T}: \mathrm{T}$ de student à $95 \%$ soit 1,96 quand $\mathrm{N}>30 ; \mathrm{N}$ : Nombre de placettes; $\mathrm{E}$ : Erreur en $\%$ sur la grandeur considérée (volume d'une essence) ; CV : Coefficient de variation en \% sur la grandeur considérée. Les formules suivantes ont été utilisées pour l'estimation des autres paramètres du plan de sondage proposés par Durrieu de Madron et Fourni (1997) :

$$
\begin{aligned}
& T_{s 1}=\left(S_{s} / S_{t}\right) \times 100 \\
& E_{q 1}=(S \times 20) / S r \\
& E_{q 2}=20 /(T s / 100) \\
& T_{s_{2}}=\left(N_{p} \times 0.5 \times 100\right) / S_{t}
\end{aligned}
$$

Avec $\mathrm{T}_{\mathrm{S} 1}$ : taux de sondage utilisé par les concessionnaires; $\mathrm{Ts}_{2}$ : taux de sondage proposé par Durrieu de Madron et Fourni; $\mathrm{E}_{\mathrm{q} 1}$ : équidistance utilisée par les concessionnaires; $\mathrm{E}_{\mathrm{q} 2}$ : équidistance proposée par Durrieu de Madron et Fourni; $\mathrm{S}_{\mathrm{s}}$ : 
superficie sondée; $\mathrm{S}_{\mathrm{t}}$ : superficie totale; $\mathrm{S}_{\mathrm{r}}$ : superficie réelle; $\mathrm{N}_{\mathrm{p}}$ : nombre de parcelles échantillonnées.

\section{Méthodologie proposée à l'administration}

Cette analyse a été faite sur la base des impacts constatés et des résultats de la simulation faite et une stratégie plus efficace a été proposée afin de pallier aux problèmes rencontrés dans l'estimation des paramètres de sondage des FC.
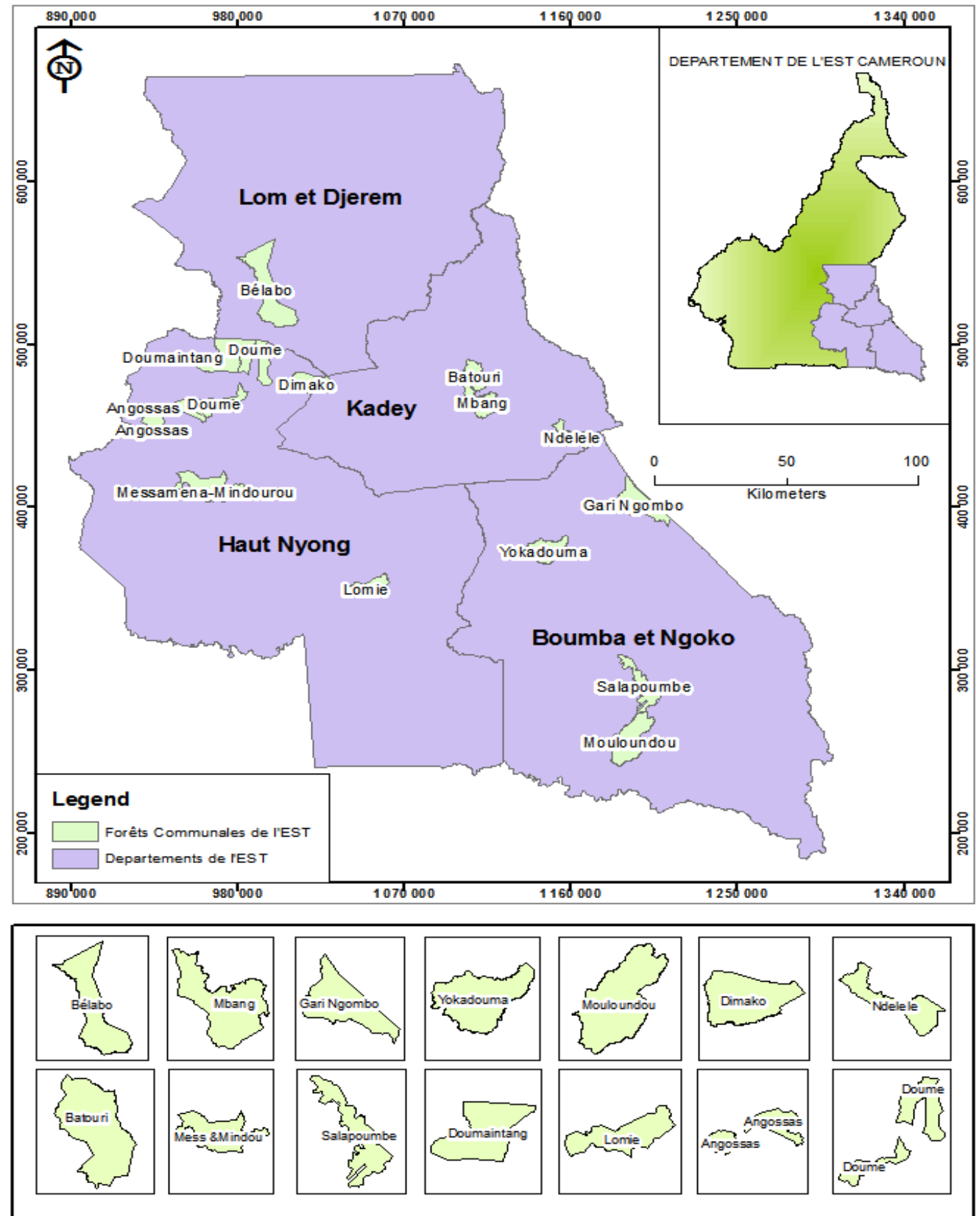

Figure 1 : Localisation des forêts communales de la région de l'Est Cameroun. 


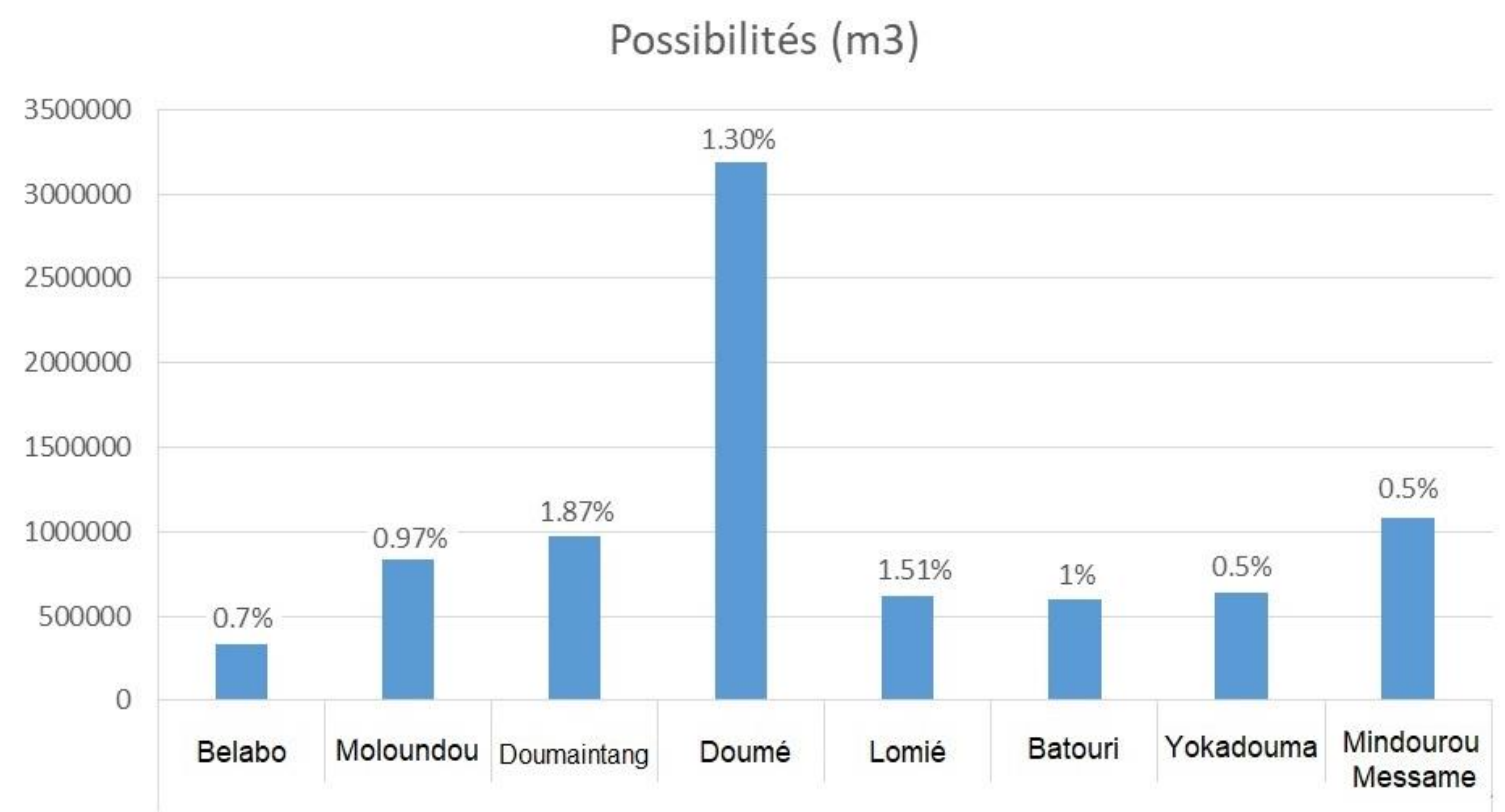

Figure 2 : Variation des possibilités en fonction des taux de sondage des FC.

\section{RÉSULTATS}

\section{État des lieux des FC de l'Est-Cameroun}

L'analyse des plans d'aménagement des $08 \mathrm{FC}$ a permis de constituer une base de données regroupant toutes les informations nécessaires pour le suivi et l'analyse de tous les paramètres d'aménagement souhaités (Tableau 1). Il présente également des données fixes préalablement définies par la loi forestière et dont l'utilisation est analogue dans chaque type de forêts communales. Parmi elles, il y a : les dégâts d'exploitation (7\%), la mortalité naturelle $(1 \%)$ et la superficie des parcelles ( $0,5 \mathrm{ha})$.

\section{Détermination de la typologie des FC en fonction du statut des plans d'aménagement et en fonction du zonage}

Le Cameroun possède un plan de zonage qui couvre une superficie de 14 millions d'hectares. Le décret $\mathrm{N}^{\circ} 95-678$ du 18 décembre 1995 détaille les dispositions janvier 1994 portant régime des forêts, de la Faune et de la Pêche notamment la subdivision du secteur forestier en un domaine forestier permanent et un domaine forestier non permanent (Tableau 2). L'existence des plans d'aménagement dans toutes les FC étudiées justifie la prise de conscience des concessionnaires sur la nécessité de gérer leur terroir avec des outils adéquats et l'intérêt de gérer durablement les forêts. Sur la base du travail de Vermeulen, le statut des forêts étudiées est celui des FC aménagées regroupées dans la zone forestière de l'Est $\mathrm{N}^{\circ} 2$ faisant l'objet d'une convention d'exploitation au profit de la communauté, car considérée comme domaine privé de celle-ci.

Les limites ou incohérences de la norme dans l'estimation des paramètres du plan de sondage des FC étudiées

L'exploitation des plans d'aménagement de chaque FC a permis de constater que l'équidistance entre les layons, le taux de sondage, le nombre de parcelles et les superficies sondées n'ont pas été donnés dans les plans d'aménagement (Doumaintang 
et Doumé). Les autres plans d'aménagement les ont cités comme référence malgré l'incohérence dans le calcul et l'implémentation de ceux-ci sur le terrain. Il a été constaté que certains paramètres comme la rotation des FC, sont uniformisés alors que les caractéristiques des forêts ne sont pas les mêmes. Le Tableau 3 présente les données des paramètres d'aménagement calculés selon la norme.

Il ressort du Tableau que l'ensemble de ces paramètres de sondage ont été calculés suivant des dispositions préétablies par la loi 94/01 du 20 janvier 1994. Compte tenu des superficies à inventorier et de la taille du massif forestier, le taux de sondage du territoire effectivement inventorié varie de $0,5 \%$ à $1 \%$ voire plus, pour les inventaires d'aménagement. C'est sur cette base qu'il a été constaté que les FC de Lomié, Doumé et Doumaintang ont des taux supérieurs à $1 \%$. Toutes ces dispositions ont permis aux concessionnaires des FC d'orienter leur aménagement suivant la norme ONADEF (1994) et de définir des formules en adéquations avec elle.

Il ressort de cette Figure 2 les différentes incohérences qui suscitent lors du choix délibéré de certains paramètres par les concessionnaires des FC. Elle démontre que Les FC de Yokadouma et Messamena appliquent le même taux de sondage (TS) et rotation dans les massifs bien qu'elles aient des possibilités différentes. La même tendance est observée entre la FC de Lomié et Doumé ayant des taux de sondage approximativement identiques, mais des possibilités significativement différentes. Ces différentes incohérences ont un impact très marqué sur les résultats d'inventaire surtout sur la gestion durable des ressources naturelles. La norme d'inventaire de préinvestissement réalisé en forêt permet l'estimation du nombre de parcelles échantillons requis pour atteindre la précision de $10 \%$ au niveau de probabilité de $95 \%$. La recherche d'une meilleure précision dans les activités d'exploitation forestière pour répondre favorablement aux objectifs de gestion durable renvoie à la prise en compte des autres méthodes notamment celle définie par Durrieu de Madron et Fourni (1997). Celle-ci stipule que pour une meilleure précision dans le calcul des paramètres d'aménagement, il est nécessaire de procéder avant toute chose à un préinventaire permettant de déterminer un coefficient de variation propre à chaque $\mathrm{FC}$.

\section{Stratégie de gestion et d'estimation des paramètres de sondage des FC par l'administration}

Pour faire un scénari et voir le comportement des paramètres de sondage en utilisant la formule de Durrieu de Madron et Fourni (2004), l'erreur (E) souhaitée a été fixée dans un premier temps à $\mathrm{E}=20$. La superficie totale utilisée dans cette analyse est la moyenne des superficies de notre échantillon estimé à : 35127,7 ha et un taux de sondage moyen 0.9. Ce qui a permis par la suite de faire varier le coefficient de variation (CV) de $150 \%$ à $350 \%$ tout en fixant l'erreur à 20 et à 25 afin d'observer comment les paramètres d'aménagement devraient se comporter.

Il ressort des Tableaux 4 et 5 que la superficie échantillonnée, le nombre de parcelles et la longueur des layons varient en fonction du coefficient de variation; tandis que l'équidistance entre les layons transversaux varie inversement au coefficient de variation. La synthèse des résultats contenus dans ces deux tableaux grâce à la méthode de calcul de Durrieu de Madron et Fourni (2004) a permis d'apprécier la variation des paramètres d'aménagement lorsque l'erreur est fixée à 20 et à 25 sur les Figures 2 et 3 . Ainsi, le Tableau 5 regroupe les taux de sondages++ des erreurs respectives 20 et 25. La Figure 4 montre que, quelle que soit l'erreur souhaitée fixée par un concessionnaire, le taux de sondage varie en 
fonction du coefficient de variation. $\mathrm{La}$ Figure 5 montre que, quelle que soit l'erreur souhaitée fixée par un concessionnaire, la superficie échantillonnée varie en fonction du coefficient de variation.

La Figure 6, quant à elle, illustre que, quelle que soit l'erreur souhaitée fixée par un concessionnaire, l'équidistance entre les layons transversaux varie inversement au coefficient de variation. On observe que le taux de sondage varie en fonction du coefficient de variation et de l'erreur souhaitée avec un impact considérable dans le nombre de parcelles échantillonnées et la longueur totale des layons transversaux. La Figure 5 présente l'évolution de la surface à échantillonner en fonction du coefficient de variation mais, qu'il n'existe pas une différence significative des superficies à échantillonner lorsque le coefficient de variation varie de 150 à 250 et l'erreur comprise entre 20 et 25 . Il ressort de la Figure 4, l'équidistance diminue au fur et à mesure que le coefficient de variation augmente et, quelle que soit l'erreur que le concessionnaire décide d'adopter le même phénomène sera observé. Ce qui aura une incidence directe sur le taux de sondage, le nombre de parcelles à échantillonner, sur la superficie à sonder et enfin sur la longueur totale des layons transversaux à définir.

\section{COURBE OMBROTHERMIQUE}

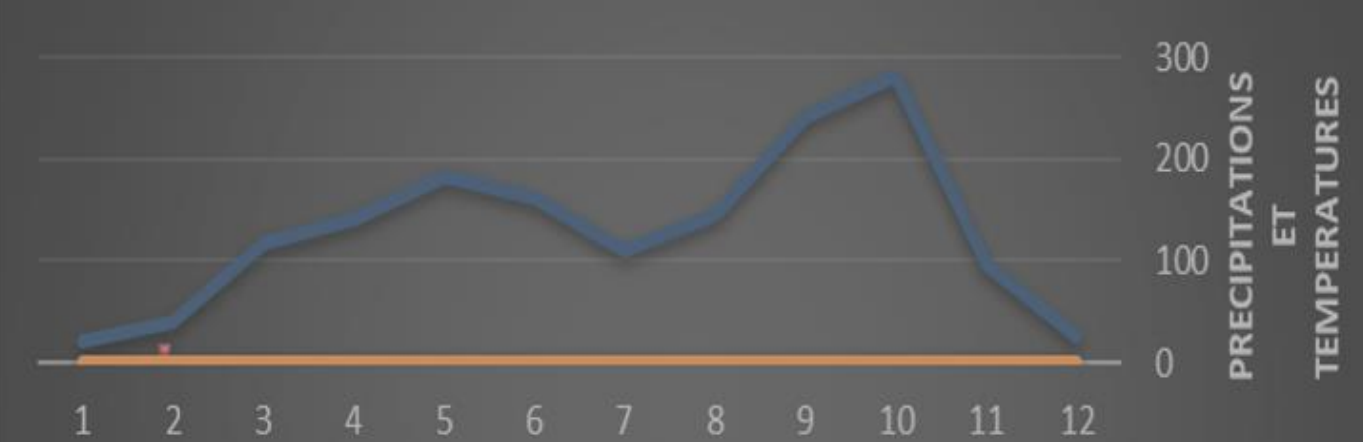

Température moyenne $\left({ }^{\circ} \mathrm{C}\right)$

- Température minimale moyenne $\left({ }^{\circ} \mathrm{C}\right)$

Température maximale $\left({ }^{\circ} \mathrm{C}\right)$

- Température moyenne $\left({ }^{\circ} \mathrm{F}\right)$

$\longrightarrow$ Température minimale moyenne $\left({ }^{\circ} \mathrm{F}\right) \longrightarrow$ Température maximale $\left({ }^{\circ} \mathrm{F}\right)$

Précipitations (mm)

Figure 3 : Courbe ombrothermique de l'Est Cameroun entre 2006 - 2010. 
A. E. BAYOI et al. / Int. J. Biol. Chem. Sci. 15(1): 169-184, 2021

Tableau 1 : Base de données des forêts communales aménagées dans la région de l'Est Cameroun.

\begin{tabular}{|c|c|c|c|c|c|c|c|c|c|c|c|c|c|c|c|}
\hline \multirow[b]{2}{*}{$\begin{array}{c}\text { Forêts } \\
\text { Communales } \\
\end{array}$} & \multicolumn{7}{|c|}{ État de la forêt } & \multicolumn{8}{|c|}{ Paramètres d'aménagement } \\
\hline & Date & $\begin{array}{c}\text { Décret de } \\
\text { Classement }\end{array}$ & $\begin{array}{c}\text { Date de } \\
\text { signature }\end{array}$ & $\begin{array}{c}\text { Superf. } \\
\text { (ha) }\end{array}$ & T.S & Equidist & Séries & AMA & Rota & $\begin{array}{l}\text { DME/ } \\
\text { AME }\end{array}$ & $\begin{array}{l}\text { S.p } \\
\text { (ha) }\end{array}$ & $\mathrm{Pr}$ & $\begin{array}{l}\text { Superf } \\
\text { sondée }\end{array}$ & $\begin{array}{c}\text { Nbre de } \\
\text { parcs }\end{array}$ & $\begin{array}{c}\text { Long } \\
\text { layons (m) }\end{array}$ \\
\hline & Aoút & $\begin{array}{l}Y=2012 \mathrm{D} \\
\mathrm{MINFOF}\end{array}$ & Août & & & & $\begin{array}{l}\text { Prod. } \\
\text { Prot. }\end{array}$ & & & & & & & & \\
\hline Belabo & 2012 & CSRRRVS & 2012 & 59213,8 & $0,7 \%$ & 2857,1 & $A G F$ & {$[0.4-0.9]$} & 30 & {$[60-100]$} & 0,5 & 332780 & 845911 & 1691,8 & 422955,5 \\
\hline Mouloundou & $\begin{array}{l}\text { Jant } \\
2006\end{array}$ & $\begin{array}{l}\mathrm{Y}=2005 \\
1475 \mathrm{PM}\end{array}$ & Mai 2005 & 42381 & $0,97 \%$ & 2061,8 & $\begin{array}{c}\text { Prod. } \\
\text { Prot. } \\
\text { Prod. }\end{array}$ & {$[0.4-0.9]$} & 30 & {$[50-100]$} & 0,5 & 837,2 & 411,1 & 822,2 & 205547,5 \\
\hline Doumaintang & $\begin{array}{l}\text { Juin } \\
2015\end{array}$ & $\begin{array}{l}N=2014 \\
3591 \text { PM }\end{array}$ & Nor: 2014 & 34718 & $1.87 \%$ & 1069.5 & $\begin{array}{l}\text { Prot. } \\
\text { AGF } \\
\text { Prod. }\end{array}$ & {$[0.4-0.9]$} & 30 & {$[50-100]$} & 0,5 & 967,9 & 649,2 & 1298,5 & 324613 \\
\hline Doumé & $\begin{array}{l}\text { Juin } \\
2015\end{array}$ & $\begin{array}{l}\mathrm{N}=2014 \\
3206 \mathrm{PM}\end{array}$ & Sept 2014 & 45359 & $1,30 \%$ & 1538,4 & $\begin{array}{l}\text { Prot. } \\
\text { AGF } \\
\text { Prod. }\end{array}$ & {$[0.4-0.9]$} & 30 & {$[60-100]$} & 0,5 & 3190,4 & 589,7 & 1179,3 & 294833,5 \\
\hline Lomié & $\begin{array}{l}\text { Avr } \\
2014\end{array}$ & $\begin{array}{l}\mathrm{N}=2010 \\
3837 \mathrm{PM}\end{array}$ & Juin 2010 & 15519 & $1,51 \%$ & 1324,5 & $\begin{array}{l}\text { Prot. } \\
\text { AGF } \\
\text { Prod. }\end{array}$ & {$[0.4-0.9]$} & 30 & {$[60-80]$} & 0,5 & 616,4 & 234,3 & 468,7 & 117168,8 \\
\hline Batouri & $\begin{array}{c}\text { Oct } \\
2014\end{array}$ & $\begin{array}{c}\mathrm{N}^{\circ} 95672 \\
\mathrm{PM}\end{array}$ & Jan: 1995 & 26203 & $1 \%$ & 2000 & $\begin{array}{l}\text { Prot. } \\
\text { AGF }\end{array}$ & {$[0.4-0.9]$} & 30 & {$[60-100]$} & 0,5 & 600,9 & 262,1 & 524,1 & 131015 \\
\hline Yokadouma & $\begin{array}{c}\text { Oct } \\
2016\end{array}$ & $\begin{array}{l}\mathrm{Y}=2005 \\
1478 \mathrm{PM}\end{array}$ & Mai 2005 & 22206 & $0,5 \%$ & 4000 & $\begin{array}{l}\text { Prod. } \\
\text { Prot. }\end{array}$ & {$[0.4-0.9]$} & 30 & {$[60-120]$} & 0.5 & 639.9 & 111.1 & 222.1 & 55515 \\
\hline $\begin{array}{l}\text { Mindourou } \\
\text { Messamena }\end{array}$ & $\begin{array}{l}\text { Aur } \\
2012\end{array}$ & $\begin{array}{l}\mathrm{N}^{\circ} 2010 \\
383 \overline{7} \mathrm{PM}\end{array}$ & Dèc 2010 & 35422 & $0.5 \%$ & 4000 & $\begin{array}{l}\text { Prod. } \\
\text { Prot. }\end{array}$ & {$[0 .-0.9]$} & 30 & {$[50-100]$} & 0.5 & 1085.7 & 708.4 & 1416.9 & 354221.5 \\
\hline
\end{tabular}

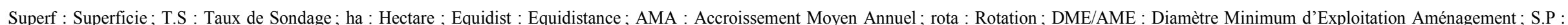

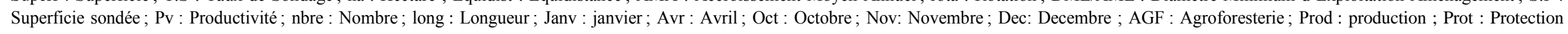


Tableau 2 : Statut juridique des forêts du Cameroun (Vermeulen, 2001).

\section{Domaine forestier permanent}

\begin{tabular}{ll}
\hline Forêts domaniales & $\begin{array}{l}\text { Forêts communales } \\
\text { Domaine privé de l'État }\end{array}$ \\
$\begin{array}{l}\text { Domaine privé de la } \\
\text { communauté }\end{array}$
\end{tabular}

\section{Domaine forestier non permanent}

\begin{tabular}{ll}
\hline Forêts communautaires & Autres forêts \\
$\begin{array}{l}\text { Démembrement du } \\
\text { domaine national }\end{array}$ & $\begin{array}{l}\text { Domaine national et } \\
\text { forêts de particuliers }\end{array}$ \\
\hline
\end{tabular}

Tableau 3 : Paramètres du plan de sondage calculés selon la norme ONADEF.

\begin{tabular}{|c|c|c|c|c|c|c|c|c|}
\hline $\begin{array}{l}\text { Forêt } \\
\text { communale }\end{array}$ & $\begin{array}{l}\text { Taux de } \\
\text { sondage } \\
(\%)\end{array}$ & $\begin{array}{l}\text { Possibilités } \\
\left(\mathbf{m}^{\mathbf{3}}\right)\end{array}$ & $\begin{array}{l}\text { Équidistance } \\
\text { layons (m) }\end{array}$ & $\begin{array}{l}\text { Superficies } \\
\text { totale (ha) }\end{array}$ & $\begin{array}{l}\text { Superficies } \\
\text { sondées } \\
\text { (ha) }\end{array}$ & $\begin{array}{l}\text { Superficie } \\
\text { parcelle } \\
\text { (ha) }\end{array}$ & $\begin{array}{l}\mathbf{N}^{\circ} \text { de } \\
\text { parcelles } \\
\text { sondées }\end{array}$ & $\begin{array}{l}\text { Longueur } \\
\text { totale } \\
\text { layon }(\mathbf{m})\end{array}$ \\
\hline Belabo & $0.7 \%$ & 332780 & 2857.1 & 59213.8 & 845.9 & 0.5 & 845.9 & 422955.5 \\
\hline Mouloundou & $0,97 \%$ & 837223 & 2061,9 & 42381 & 411,1 & 0,5 & 411,1 & 205547,5 \\
\hline Doumaintang & $1,87 \%$ & 967963 & 1069,5 & 34718 & 649,2 & 0,5 & 649,2 & 324613 \\
\hline Doumé & $1,3 \%$ & 3190390 & 1538,5 & 45359 & 589,7 & 0,5 & 589,7 & 294833,5 \\
\hline Lomié & $1,51 \%$ & 616380 & 1324,5 & 15519 & 234,3 & 0,5 & 234,3 & 117168,8 \\
\hline Batouri & $1 \%$ & 600998 & 2000 & 26203 & 262,1 & 0,5 & 262,1 & 131015 \\
\hline Yokadouma & $0,5 \%$ & 639991 & 4000 & 22206 & 111,1 & 05 & 111,1 & 55515 \\
\hline $\begin{array}{l}\text { Mindourou } \\
\text { Messamena }\end{array}$ & $0,5 \%$ & 1085721 & 4000 & 35422 & 708,4 & 0,5 & 708443 & 354221,5 \\
\hline
\end{tabular}

Tableau 4 : Variation des paramètres d'aménagement à une erreur fixée à 20.

\begin{tabular}{llllllll}
\hline $\mathbf{C V}$ & $\mathbf{E r}$ & $\mathbf{N p}$ & Ts (20) & St & Se (20) & Eq (20) & Ltl \\
\hline 150 & 20 & 216,09 & 0,307577562 & 35127725 & 108045 & 6502,42492 & 54022,5 \\
200 & 20 & 384,16 & 0,546804554 & 35127725 & 192,08 & 3657,61401 & 96040 \\
250 & 20 & 600,25 & 0,854382116 & 35127725 & 300125 & 234,87297 & 150062,5 \\
300 & 20 & 864,36 & 1,230310246 & 35127725 & 432,18 & 1625,60623 & 216090 \\
350 & 20 & 1176,49 & 1,674588946 & 35127725 & 588245 & 1194,32294 & 294122,5
\end{tabular}

$\overline{\mathbf{C V}}$ : coefficient de variation, Er : erreur, Np : parcelle échantillon, Ts : taux de sondage, St : surface totale moyenne, Se : surface échantillonnée, Eq : équidistance, Ltl : longueur totale des layons. 
Tableau 5 : Variation des paramètres d'aménagement à une erreur fixée à 25.

\begin{tabular}{llllllll}
\hline $\mathbf{C V}$ & $\mathbf{E r}$ & $\mathbf{N p}$ & Ts (25) & St & Se (25) & Eq (25) & Ltl \\
\hline 150 & 25 & 138.2976 & 0,196849639 & 35127725 & 69,1488 & 10160,0389 & 34574,4 \\
200 & 25 & 245.8624 & 0,349954915 & 35127,25 & 122.9312 & 5715,0219 & 61465,6 \\
250 & 25 & 384,16 & 0,546804554 & 35127,25 & 192,08 & 3657,61401 & 96040 \\
300 & 25 & 553.1904 & 0,787398558 & 35127725 & 276.5952 & 2540,00973 & 138297,6 \\
350 & 25 & 752.9536 & 1,071736926 & 35127725 & 376.4768 & 1866,1296 & 188238,4
\end{tabular}

$\overline{\mathbf{C V}}$ : coefficient de variation, Er : erreur, Np : parcelle échantillon, Ts : taux de sondage, $\mathbf{S t}:$ surface totale moyenne, Se : surface échantillonnée, $\mathbf{E q}$ : équidistance, $\mathbf{L t l}$ : longueur totale des layons.

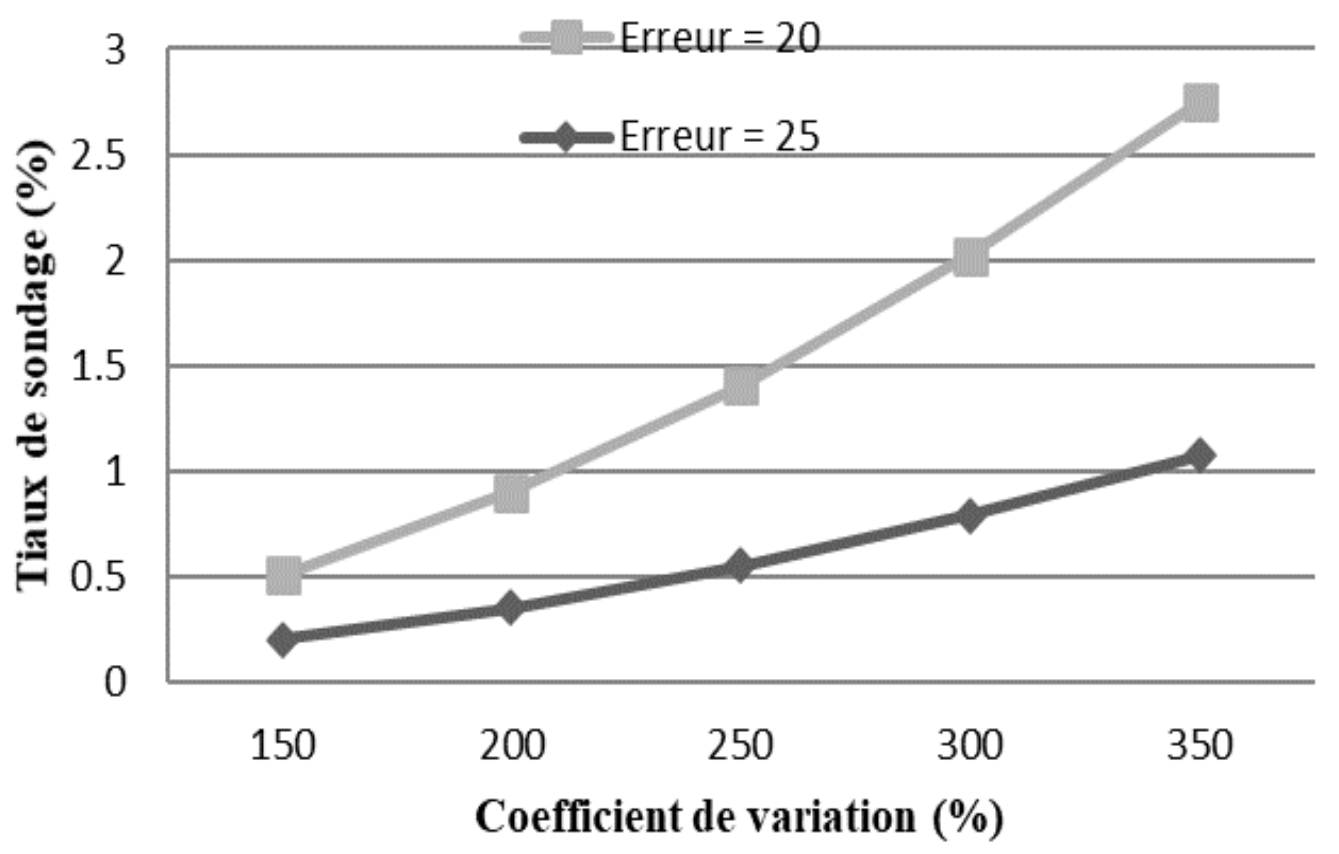

Figure 4 : Variation du taux de sondage en fonction de l'erreur et du coefficient de variation. 


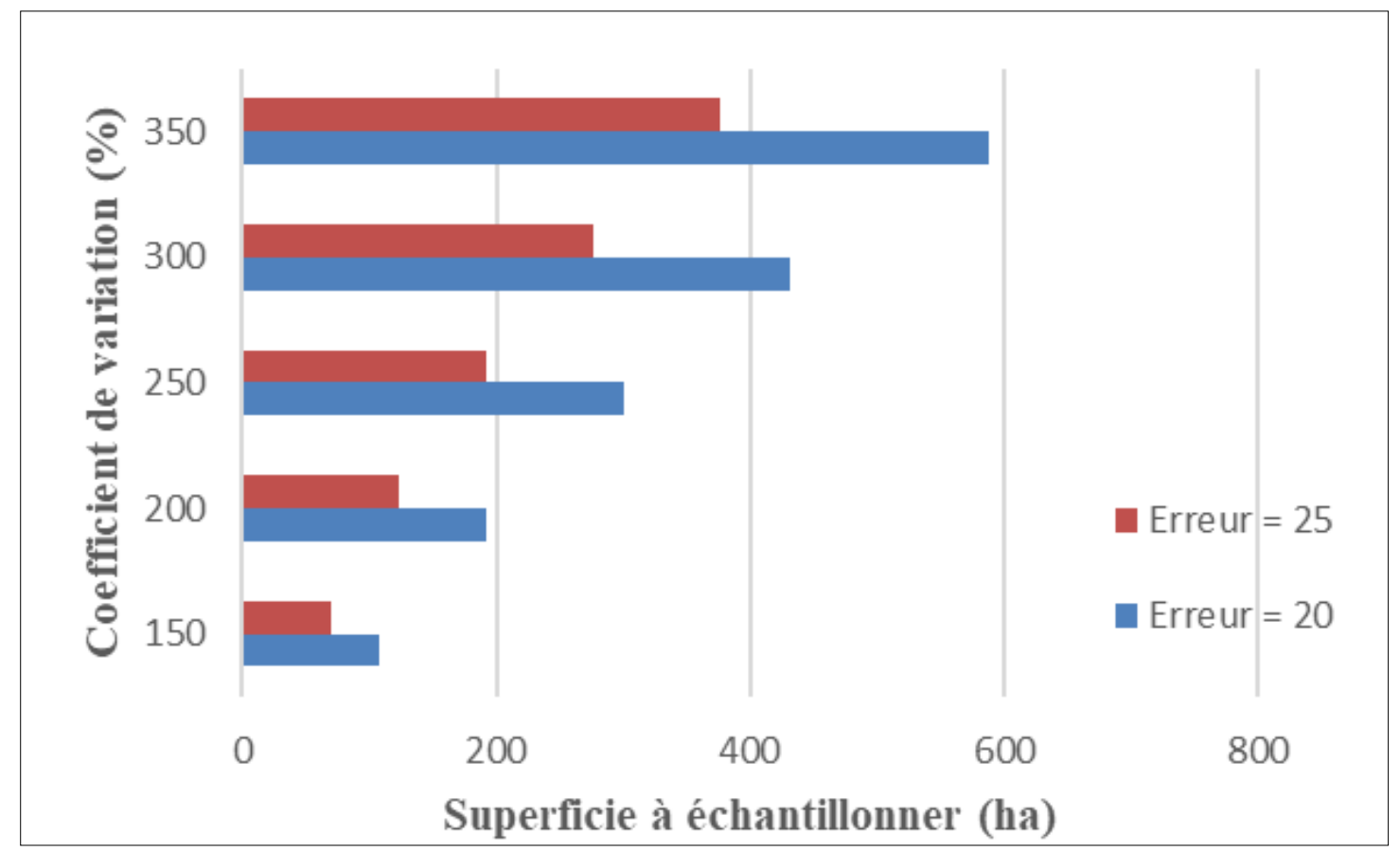

Figure 5 : Variation du coefficient de variation en fonction de la superficie à échantillonner.

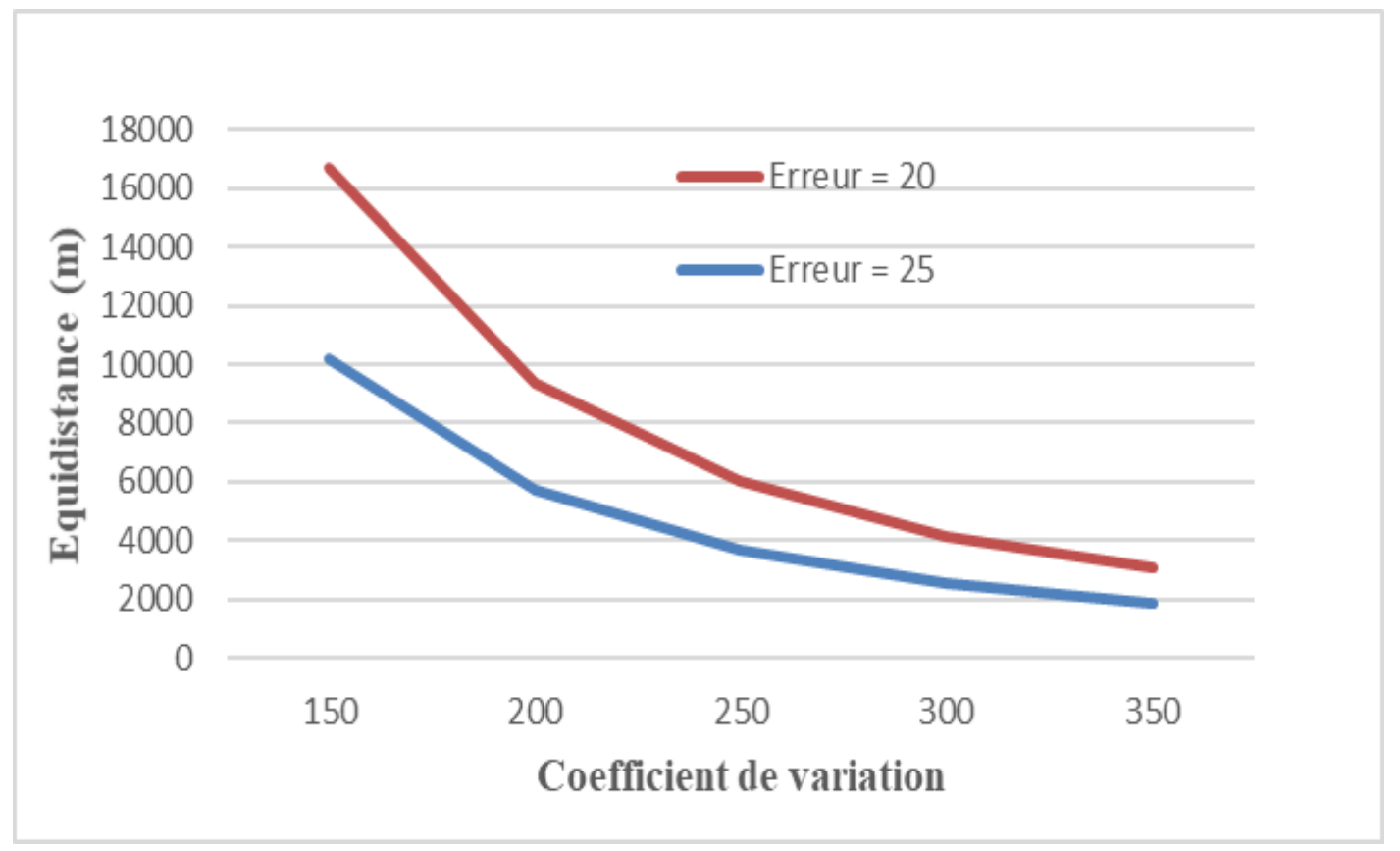

Figure 6 : Variation de l'équidistance en fonction de l'erreur et du coefficient de variation. 


\section{DISCUSSION}

\section{Taux de reconstitution des FC}

Les superficies et production de chaque FC étudiées sont différentes, la rotation adoptée dans les plans d'aménagement est similaire: de 30 ans ou 30 ans plus un multiple de cinq selon la loi 94/01 du 20 janvier 1994. Ce qui suscite un questionnement sur ce choix arbitraire des concessionnaires et la capacité des FC à se reconstituer de manière durable, également celle de certaines essences spécifiques. La durée optimale de rotation serait celle pour laquelle on obtient le meilleur taux de reconstitution du nombre de pieds ou du volume prélevés (Terreaux et al., 2011). L'Ayous par exemple a été classé première essence exploitée au Cameroun en 2016 et 2017 (COMCAM, 2017). Par ailleurs, les incohérences observées dans les plans d'aménagement en ce qui concerne le taux de sondage observées entre autres dans les FC de Batouri et Doumaintang par exemple peuvent entraîner un problème de régénération à plus ou moins long terme. Wilson (2013) a observé après avoir vérifié plus de 2000 calculs de reconstitution que presque la moitié $(47 \%)$ était incorrecte et souvent considérablement surestimée. De plus, le taux de reconstitution augmente avec la durée de la rotation. Les mêmes manquements ont également été observés sur les équidistances entre les layons ce qui remet en question la qualité des plans d'aménagement des FC au Cameroun. D'après le même auteur, le caractère réellement durable des réformes entamées depuis le milieu des années 90 est encore loin d'être acquis. D'autres FC bien que situées à des zones distinctes et avec des superficies différentes avaient également des taux de reconstitution similaires pourtant, le taux de reconstitution dépend de la localisation de la valeur modale de la distribution par rapport à la valeur du DME prélevé (Terreaux et al., 2011). Ce qui prouve que ces plans d'aménagement n'ont pas pris en compte les conditions spécifiques du milieu.
Typologie des FC suivant le statut des plans d'aménagement et du zonage

Les forêts communales étudiées dans la région de l'Est Cameroun ont chacune un plan d'aménagement approuvé par l'administration en charge des forêts et occupent une superficie de 281021,8 ha soit environ $31224 \%$ de la superficie nationale classée. Ce qui veut dire qu'en 10 ans d'exploitation ce bloc a été achevé et un volume de $14271446 \mathrm{~m}^{3}$ de matière du peuplement résiduel a disparu. Compte tenu de ce taux de déforestation élevé, des mesures de gestion devraient être mises en place afin de restaurer les zones exploitées et protéger la superficie restante (616 970,2 ha). Malheureusement, on se rend compte que les techniques d'aménagement utilisées dans ses plans d'aménagement correspondent plus à la réalisation de plans d'exploitation qu'à celle de véritables aménagements forestiers. L'insuffisance de ressources financières est la cause de la nonréalisation de ses études préalables. Les administrations forestières doivent ainsi établir des mesures d'accompagnement des FC et s'assurer de l'application effective des plans d'aménagement. Aujourd'hui bien que l'aménagement forestier a progressé au Cameroun (Samyn et al., 2011), la conception et la mise en œuvre de ces plans ne respectent pas pour une forêt exploitée: le renouvellement de la ressource ligneuse; la préservation de la biodiversité; et les aspects de développement socio-économique et participatif.

\section{Impact du non-respect des normes}

L'analyse des résultats a révélé que l'importance de commencer les activités d'exploitation forestière par un préinventaire servira à définir un coefficient de variation spécifique au massif forestier (Ngibaot and Leersum, 2000). Cette variable augmente la précision et apporte une meilleure cohérence dans le calcul d'autres paramètres de sondage. En effet, le taux de sondage varie en fonction $\mathrm{du}$ coefficient de variation et de l'erreur souhaitée avec un impact considérable dans le nombre de parcelles échantillonnées et la longueur totale des layons transversaux. Par 
ailleurs, il n'existe pas une différence significative des superficies à échantillonner lorsque le coefficient de variation varie de 150 à 250 et l'erreur comprise entre 20 et 25 . On devrait s'attendre alors à une diminution de l'équidistance au fur et à mesure que le coefficient de variation augmente. Il est clair que cette étape préalable peut contribuer de façon significative à la réduction du problème de sur ou sous-estimation du nombre de parcelles et contribuer à une meilleure planification des activités d'exploitation. Les coûts prévisionnels des gestionnaires de l'aménagement des forêts communales devront également être positivement affectés.

\section{Impacts de l'utilisation des paramètres de sondage erronés sur les inventaires}

Le potentiel existant pourrait être mal distribué, car la non-actualisation des inventaires nationaux entraîne une sous ou surestimation du potentiel existant sur une superficie donnée. La surestimation des parcelles qui augmente le coût des activités d'exploitation forestière peut entraîner ainsi la faillite et la fermeture de ses organismes (Borie, 2000). Une mauvaise estimation des paramètres de sondage pourrait aboutir à une surexploitation de la forêt provoquant ainsi la disparition de la biodiversité. Pourtant les données de mortalité et d'accroissement sont également capitales pour le calcul des paramètres d'aménagement (Bayol and Borie, 2004). En outre, la surexploitation des ressources pour la satisfaction des besoins actuels si l'exploitation est maintenue comme pratiquée de nos jours ne pourra pas garantir celle des générations futures. Comme le note Durrieu de Madron (2004), le plan d'aménagement forestier n'est pas suffisamment considéré ou compris comme un outil de gestion, de programmation à court et moyen terme et de rationalisation dans l'espace et dans le temps de l'exploitation. La conception de ce document est plus perçue comme une étape du cahier de charges et apparaît dès lors comme une simple étape administrative nécessaire à l'obtention de la convention définitive. Par ailleurs, la démarche d'aménagement passe en général après les préoccupations liées à la production du bois (Esteve, 2001). D'après certains experts, l'exploitation des paramètres non contrôlés a des impacts plus graves sur les pertes de forêts, car elle dégrade progressivement les forêts situées à proximité des zones densément peuplées (Megevand et al., 2013). Le principe qui prévaut dans ce cas est celui d'une planification de l'aménagement très simplifiée (Samyn et al., 2011). Il revient dès lors à l'administration forestière de mettre en place des mesures cohérentes et réalistes.

\section{Conclusion}

La présente étude avait pour objectif de contribuer à la gestion durable des ressources forestières en général et ligneuses en particulier au travers de l'effectivité des plans d'aménagement des forêts communales. Il ressort de là que les forêts communales échantillonnées fonctionnent principalement sur la base de leur plan d'aménagement évalué et approuvé par l'administration responsable des forêts. Toutefois, face à l'incohérence dans le calcul des différents paramètres de sondage observés (équidistance et le taux de sondage), la gestion forestière au Cameroun offre toujours théoriquement un cadre performant de gestion des ressources forestières. Malheureusement, son implémentation impose aux gestionnaires des coûts importants qu'ils ne peuvent difficilement absorber, d'autant plus que l'amortissement de ces coûts se fait sur une échelle de temps qui dépasse souvent leur propre perspective économique. Ce facteur est une des causes majeures qui limite la mise en œuvre de ses principes de gestion durable des ressources forestières. Bien que des efforts ont été faits, l'élaboration des plans d'aménagement dans les forêts communales reste une menace qui pèse sur ces écosystèmes. Des mesures d'aménagement plus réalistes qui s'appuient sur des considérations économiques et sociales doivent être mises en place notamment en ce qui concerne la durée de la rotation, la détermination des diamètres d'exploitabilité et 
la cohérence dans le calcul des paramètres de sondage.

\section{CONFLITS D'INTÉRÊTS}

Les auteurs ne déclarent aucun conflit d'intérêts.

\section{CONTRIBUTIONS DES AUTEURS}

BEA et EMB ont rédigé le premier draft et préparé les résultats. BEA et MJYN ont révisé et préparé le document final.

\section{REMERCIEMENTS}

Les auteurs remercient tout le personnel et corps enseignant du DEPFOR, notamment Pr. BOBO KADIRI SERGE et Pr Martin TCHAMBA pour leurs conseils, appuis techniques, encouragements et disponibilité lors de la réalisation de cette étude

\section{RÉFÉRENCES}

Bayol N, Borie JM. 2004. Itinéraires techniques d'aménagement des forêts de production en Afrique centrale. Bois et Forêts des Tropiques, 281(3) : 35-49. DOI :

https://doi.org/10.19182/bft2004.281.a20 225

Borie JM. 2000. Propositions d'amélioration des paramètres d'aménagement, projet Forêts et Terroirs. Actes de l'atelier de restitution, juin 2000.

Bobo KS. 2020. Bilan diagnostic de l'état de la «biodiversite » dans quelques UFA du Sud Cameroun (UFA 10-018, 10-015, $10-063,10-011,10-012,10-047$ et 10037).

CENADEFOR. 1983. Inventaire des ressources forestières du Sud Cameroun (Iére tranche). Fascicule II Résultats. CENADEFOR.

COMCAM. 2017. Rapport COMCAM 2017. Banque de données du MINFOF relative à l'exportation de bois à partir de Douala. Ministère des forêts et de la faune, Commerce du bois au Cameroun (COMCAM), Yaoundé.

COMIFAC. 2007. Programme d'action sousrégionale de lutte contre la dégradation des terres et la désertification en Afrique centrale (pasr/lcd-ac)

Cuny P. 2001. État des lieux de la foresterie communautaire et communale au Cameroun. Tropenbos internattional programme du bassin du Congo. $11 \mathrm{p}$.

Durrieu de Madron LD, Forni E. 2004. L'arbitraire dans l'aménagement en zone tropicale, ses justifications et sa gestion. Séminaire CIRAD sur le Bassin du Congo. 23 p.

Durrieu de Madron LD, Forni E, Mekok M. 1998. Les techniques d'exploitation à faible impact en forêt dense humide camerounaise. Serie FORAFRI No. Document 17. Montpellier, France, CIRAD-Foret. $\quad 30 \quad$ p. ; http://hdl.handle.net/10568/18037.

Durrieu de Madron LD, Forni E. 1997. Aménagement forestier dans l'Est du Cameroun. Structure du peuplement et périodicité d'exploitation. Bois et Forêt des Tropiques, 254: 39-50. http://agritrop.cirad.fr/389267/1/docume nt_389267.pdf

Esteve J. 2001. Étude sur le Plan pratique d'aménagement des forêts naturelles de production tropicales africaines, application au cas de l'Afrique centrale. Premier volet: Production forestière. Paris, France, ATIBT, 91 p.

FAO. 2003. Code régional d'exploitation forestière à faible impact dans les forêts denses tropicales humides d'Afrique centrale et de l'Ouest. Rome. 152 p.

FAO. 2018. Rapport sur l'état de la transparence dans le secteur forestier en Afrique centrale. $2 \mathrm{p}$

Gonmadje CF, Donfack JJ. 2015. L'état des ressources génétiques forestières mondiales. Rapport national du Cameroun.

Kengoum Djiegni F, Assembe MS, Atyi R, Eba'a Levang P, Fomete T. 2016. Les politiques forestières et le contexte national de l'utilisation des terres au Cameroun: Des Approches sectorielles vers une cohérence globale? The International Forestry Review, 18. 
Lescuyer G, Cerutti PO, Tsanga R. 2016. Contributions of community and individual small-scale logging to sustainable timber management in Cameroon. International Forestry Review, 18: 40-51. DOI: $10.1505 / 146554816819683744$.

Letouzey R. 1985. Notice de la carte phytogéographique du Cameroun au 1 : 500000 . Institut de la carte internationale de la végétation, Toulouse. Megevand C, Aline M, Joël H, Klas S, Nina D, Charlotte S. 2013. Dynamiques de déforestation dans le bassin du Congo réconcilier la croissance économique et la protection de la forêt. The World Bank. DOI : 10.1596/978-0-8213-97428.

MINFOF. 2011. Aménagement forestier. Sous-Direction des Inventaires et de l'Aménagement Forestier (SDIAF).

MINFOF. 1994. Diagnostic de la situation de l'environnement de la province du Sud. Élaboration du plan - national de gestion de l'environnement au Cameroun. Cellule de coordination du PNGE, projet PNUD CMR/921008, Banque Mondiale et GTZ, $145 \mathrm{p}$. tannexes.

MINFOF. 2015. Secteur forestier et faunique du Cameroun : faits et chiffres. Finaliser l'accord de partenariat volontaire au Cameroun.

Mertens B, Steil M, Ayenika L, Nebashu G, Minnemeyer S. 2007. Atlas forestier interactif $\mathrm{du}$ Cameroun. Version 2.0. Washington D.C. World ressource Institute. $48 \mathrm{p}$.

Ngibaot F, Leersum GV. 2000. Inventaire forestier d'exploitation, pour quelles précisions? Kribi-Cameroun. http:www.Pefac.com/pdf/Ngibaot.Pdf

Ngoufo R, Nouhou N, Parren M. 2012. État des lieux de la situation économique, écologique et sociale actuelle de l'espace camerounais du TRIDOM. Tropenbos International - Programme du bassin du Congo, Wageningen, Pays-Bas. X +145 pp.

OAB/OIBT. 2005. Manuel d'audit pour la mise en œuvre des principes, critères et indicateurs OAB-OIBT de gestion durable des forêts tropicales naturelles d'Afrique au niveau de l'UFA. Version finale. Septembre 2005. $46 \mathrm{p}$.

OIBT. 2006. Actualités des forêts tropicales (édition spéciale). Situation de l'aménagement des forêts tropicales en 2005 : Résumé analytique. $36 \mathrm{p}$

ONADEF. 1994. Canevas de plan d'aménagement et de préinvestissement. Ministère d'Agriculture (MINAGRI), Yaoundé, Cameroun. Annexes, 7 p,

PFBC. 2006. Les forêts du bassin du Congo. Rapport 2006 sur l'état des forêts. RDC, 258p.

Samyn JM. 2011. Secteur forestier dans les pays du Bassin du Congo: 20 ans d'interventions de l'AFD. Intercooperation (IC), Suisse James GASANA, IC Emmanuel POUSSE, Institutions et Développement (I\&D), France Fabien POUSSE, I\&D.

Terreaux JP, Weber J. 2011. Réflexions sur l'évaluation de la biodiversité et l'aménagement des forêts. Revue Forestière Française, 63 (5) : 571-578. DOI : $10.4267 / 2042 / 46105$.

Wilson M. 2013. Calculs des diamètres minimums d'exploitation durable : méthode prenant en compte le type de forêt et l'historique d'exploitation au Cameroun. 